\title{
Temporal and dynamic changes in plantar pressure distribution, as well as in posture during slow walking in flat and high-heel shoes
}

\author{
SONA JANDOVA ${ }^{1 *}$, MILOSLAV GAJDOŠ ${ }^{2}$, \\ KATARÍNA URBANOVÁ ${ }^{2,3}$, WiOLETTA MIKULÁKOVÁ ${ }^{2}$ \\ ${ }^{1}$ Technical University of Liberec, Faculty of Mechanical Engineering, \\ Department of Applied Mechanics, Liberec, Czech Republic. \\ ${ }^{2}$ University of Prešov in Prešov, Faculty of Health Care, Department of Physiotherapy, Prešov, Slovakia. \\ ${ }^{3}$ St. Elizabeth College of Health and Social Work, Bratislava, Slovakia.
}

\begin{abstract}
Purpose: The aim of the study was to assess the differences in plantar pressure distribution and in posture during slow and very slow walking on the treadmill in flat and high-heeled shoes. Methods: The Pedar- $\mathrm{X}^{\circledR}$ (Novel, Munich, Germany) measuring insoles were used to measure the plantar pressure distribution and to assess temporal parameters during walking on the treadmill with the speed $v_{1}=0.97 \mathrm{~ms}^{-1}$ and $v_{2}=0.56 \mathrm{~ms}^{-1}$ in flat shoes (FS) and HH. For postural measurement, the SonoSens Monitor Analyzer (Gefremed, Chemnitz, Germany) was used. Thirty healthy females who only occasionally wear HH volunteered to participate in the study (age: $21.8 \pm 2.09$ years, weight: $55.7 \pm 4.05 \mathrm{~kg}$, height: $1.66 \pm 0.03 \mathrm{~m})$. For statistical analysis the ANOVA, and paired $t$-test were used. Results: Significant differences in walking in $\mathrm{HH}$ were detected in temporal and plantar pressure variables, compared to FS. The walking speed influenced the temporal variables, but not the measured dynamic parameters, in either shoes. In the sagittal plane, significant differences in all sections of the spine were identified for $v_{1}$ and $v_{2}$. While walking in $\mathrm{HH}$, lordosis at lumbar spine decreased significantly as well as at cervical spine, and kyphosis increased in the thoracic spine. In HH statistically significant differences between the walking speeds were observed particularly in the thoracic spine. Conclusions: Walking in high heels caused a plantar pressure changes and curvature of the spine, and the slowing of walking speed did not prevent these changes.
\end{abstract}

Key words: gait cycle, stance, swing, frontal plane, sagittal plane

\section{Introduction}

Gait is the basic movement stereotype and motor expression of an individual, and one of the basic features of the human form. In terms of natural human movement, the choice of footwear is very important. In our modern society, women often wear high-heeled shoes $(\mathrm{HH})$ which cause significant changes in both the dynamics and kinematics of gait, power profile, and motor control [5]. HH are defined as shoes whose heel is located higher than its tip; the difference can be more than $10 \mathrm{~cm}$ [6]. In comparison, flat shoes (FS) enable the full range of take-off and longer strides are, therefore, possible when increasing walking speed. The Centre of Mass (CoM) places the load of the foot at the Chopart's joint area, which makes upright walking easier in FS. Similarly to tiptoeing, while walking in $\mathrm{HH}$, the $\mathrm{CoM}$ shifts forward to the Lisfranc joint area. It increases the pressure on the metatarsal area, and the load is removed from the heel [3], [18]. The kinetic effects of $\mathrm{HH}$ also depend upon the habit of their wearer, their age and walking speed [19], [24].

During the observation of changes in the plantar area caused by wearing different types of shoes, the plantar pressure distribution and Centre of Pressure $(\mathrm{CoP})$ are evaluated. Besides other factors, the $\mathrm{CoP}$

\footnotetext{
* Corresponding author: Sona Jandova, Applied Mechanics, Technical University of Liberec, Faculty of Mechanical Engineering, Studentská 2, 46008, Liberec, Czech Republic. Tel: +420 485353656, e-mail: sona.jandova@tul.cz

Received: August 7th, 2019

Accepted for publication: October 22nd, 2019
} 
also depends on the profile and tilt of the supporting surface as well as the friction properties of both the surface and the shoes [10]. While walking, the CoP usually shifts from the centre of the heel, along the external side of the foot towards the fifth metatarsal, and from the tips of the toes towards the big toe. $\mathrm{HH}$ prevent the wearer from stepping on their heel properly and landing on the ground gradually because the foot is in plantar flexion. The higher the heel, the more the frontal part of the foot is overloaded [3], [4], [30], increasing the pressure and duration of pressure under the medial forefoot during walking [30], [3]. In $\mathrm{HH}$, the stance phase is extended, which increases the duration of the contact between the foot and the surface [2]. $\mathrm{HH}$ influence the walking speed and shorten the steps [2], [7], [9], [16]. The main characteristics of gait include the gait cycle duration, rhythm, stride, and speed [28], [13], [14]. Walking speed is limited by frequency and stride; the latter depends upon the extent of the lower limb range of motion during the swing phase. Walking speed changes during the gait cycle and it is influenced by the position of the stance of the lower limb. When the foot is in front of the body, the walking speed decreases. When the foot is behind the body, the walking speed increases. The average walking speed of an adult is $1.33 \mathrm{~m} \cdot \mathrm{s}^{-1}-1.53 \mathrm{~m} \cdot \mathrm{s}^{-1}[18]$.

$\mathrm{HH}$ contribute to slower self-selected walking speeds and shorter strides, whereas cadence is generally unchanged [2], [7], [9]. Studies examining various attributes of walking in $\mathrm{HH}$ most often used a walking speed of 1.3-1.4 $\mathrm{m} \mathrm{s}^{-1}$ [2], [26]. A slow speed is considered to be approx. $1.12 \mathrm{~m} \mathrm{~s}^{-1}$ [17]. This values seem to be still relatively high for those who have little experience with wearing $\mathrm{HH}$.

$\mathrm{HH}$ also influence the overall postural stability. The foot position causes a chain effect influencing the position of the lower limbs, pelvis, and spine [6]. Walking in $\mathrm{HH}$ also depends upon the wearer's experience with $\mathrm{HH}$ [19], [5], experienced wearers (wearing $\mathrm{HH}$ approximately 4 hours/day, 4 days/week or more) exhibited better control during the contact phase of one-limb support as well as a faster transfer of weight during double-limb support. The previous study [19] showed that, while walking in $\mathrm{HH}$, the lumbar area lordosis straightened in women who do not wear $\mathrm{HH}$ or do so rarely, however, it was more pronounced in experienced $\mathrm{HH}$ wearers.

The postural and locomotion motor skills interact to ensure coordination and fluent movement; they are activated automatically. However, the discrepancy caused between the new type of movement and postural motor skills in inexperienced wearers causes them to decrease their walking speed. Therefore, it seems important to focus on very slow walking speed in $\mathrm{HH}$ (at $<1 \mathrm{~m} \mathrm{~s}^{-1}$ ), typical for the inexperienced $\mathrm{HH}$ wearers. No study on very slow walking seems to be available. The main aim of presented study is to find out whether very slow walking $\left(v<1 \mathrm{~m} \mathrm{~s}^{-1}\right)$ observed in a group of inexperienced $\mathrm{HH}$ wearers significantly changes the dynamic characteristics and whether it causes postural changes as occur during higher walking speeds (as discovered in previous studies). It is assumed that slow walking in $\mathrm{HH}$ will increase the $\%$ duration of the STANCE phase, while the SWING phase will be relatively shorter than while walking in FS. It is expected that ground reaction forces (GRF) in the forefoot while walking in $\mathrm{HH}$ will occur as well as postural changes in the sagittal plane represented by straightening of cervical and lumbar lordosis, and thoracic kyphosis.

\section{Materials and methods}

\section{Participants}

Thirty healthy females who wear HH only occasionally volunteered to participate in the study (age: 21.8 \pm 2.09 years, weight: $55.7 \pm 4.05 \mathrm{~kg}$, height: 1.66 \pm 0.03 m, BMI: $20.34 \pm 1.41$, shoe size: EU 36-38). The exclusion criteria included previous musculoskeletal injury within 1 year and experiencing musculoskeletal pain; none of the participants experienced any injuries that could limit their range of motion of their body and extremities. The study was approved by the Institutional Review Board for testing of human subjects (protocols were obtained under the license granted by this board) and the research was performed in accordance with the ethical standards of the Helsinki Declaration.

\section{Data collection}

The Pedar- $\mathrm{X}^{\circledR}$ (Novel, Munich, Germany) insole measuring system was used to measure the plantar pressure distribution and to assess temporal and dynamic parameters during the gait cycle: 30 -s walk in flat shoes (FS) and 30-s walk in high heel shoes (HH). This measuring system enables data to be recorded with a frequency of $100 \mathrm{~Hz}$. The data collected were analysed with the Pedar- $X^{\circledR}$ (Novel, Munich, Germany) software after the measurements were finished. The subjects walked on a treadmill (inSPORTline Genesis, Slovakia) at the pre-set walking speeds of $v_{1}=0.97 \mathrm{~m} \cdot \mathrm{s}^{-1}$ (slow speed) and $v_{2}=0.56 \mathrm{~m} \cdot \mathrm{s}^{-1}$ (very slow speed). From the participants' point of view, these speeds were perceived as the safest and most comfortable. Speed $v_{1}$ was perceived as comfortable slow speed, $v_{2}-$ as very slow. Shoes and speeds were selected randomly. 
For postural measurement, the SonoSens Monitor Analyzer system (Gefremed, Chemnitz, Germany) was used to observe the movements in individual sections of the subjects' spines as well as their posture when walking. In a comparative experiment, the correlation between the variables recorded when walking in two types of shoes were identified.

\section{Protocol}

Data pertaining to each subject during walking were obtained during a single session. The participants were first familiarized with the experimental protocol and subsequently signed an informed consent form. At the beginning of the data collection, anthropometric data were recorded. The participants' height was measured using a portable Antropometer A 213 (Trystom, Olomouc, Czech Republic), their weight was measured using an electronic scale (Amboss, New York, USA), and their age in years was recorded.

At the beginning of each trial, the measuring insoles were calibrated by standing on one leg separately for each shoe, in accordance with the manufacturer's recommendations.

Two types of shoes (Fig. 1) were used (FS and HH shoes with a $7 \mathrm{~cm}$ heel and $1.4 \times 1.2 \mathrm{~cm}$ heel thickness) for the measurement and subjects chose their respective shoe size. Subjects were asked to walk on the treadmill in both types of shoes for 3 minutes before the test started. At first, all participants walked in randomly chosen shoes at the pre-set walking speed $\left(v_{1}=0.97 \mathrm{~m} \cdot \mathrm{s}^{-1}\right.$ and $\left.v_{2}=0.56 \mathrm{~m} \cdot \mathrm{s}^{-1}\right)$. The measurement for the other type of shoes (identical $v_{1}$ and $v_{2}$ ) continued after a 5-minute rest during which the recalibration of the system was performed. All subjects were tested in the same type of shoes of the respective size.

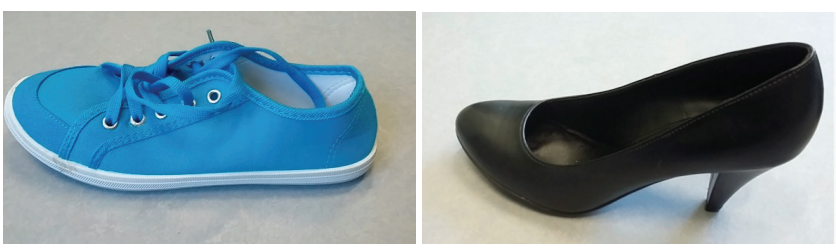

Fig. 1. Flat shoes (left) and high heel shoes (HH) used in the study

During the postural measurement, the measuring system was first calibrated during upright standing, forward bending and backward bending of the spine, side bending and rotation in both directions as recommended by the Sonosens Monitor Analyzer manufacturer. The movements of the axial skeleton during gait analysis are represented by $\%$ deviations from the default data measured during the calibration.

\section{Plantar pressure analysis}

In the analysis of the gait cycle, temporal and dynamic variables were measured and calculated (Fig. 2). From the measured maximal peak pressure values $\left(P P_{\max }\right)$ and maximal vertical force $\left(F_{\max }\right)$ functions, the following temporal and dynamic variables for each step were observed: The STANCE [s] phase - which represents the contact time with the ground, SWING [s] phase and duration of the STRIDE [s], frequency (FRE) [steps/min], maximal peak pressure per body weight $\left(P P_{\operatorname{maxBW}}=P P_{\max } / \mathrm{m} \cdot \mathrm{g}\right)$ and maximal vertical force per body weight $\left(F_{\operatorname{maxBW}}=F_{\max } / \mathrm{m} \cdot \mathrm{g}\right)$.

Thirty steps from the central part of the 30 -s measurement section were evaluated. The first $6-8$ and the last 6-8 steps were recorded, but not evaluated due to the necessity to evaluate only the fluent walking cycle. The resulting data pertaining to left and right feet are the mean values calculated based on 15 steps using each leg. The number of steps for the analysis was based on the individual length of the step on the measured section. The coefficients of variation $(\mathrm{CVs})$ pertaining to the calculated mean values were $1-3 \%$.

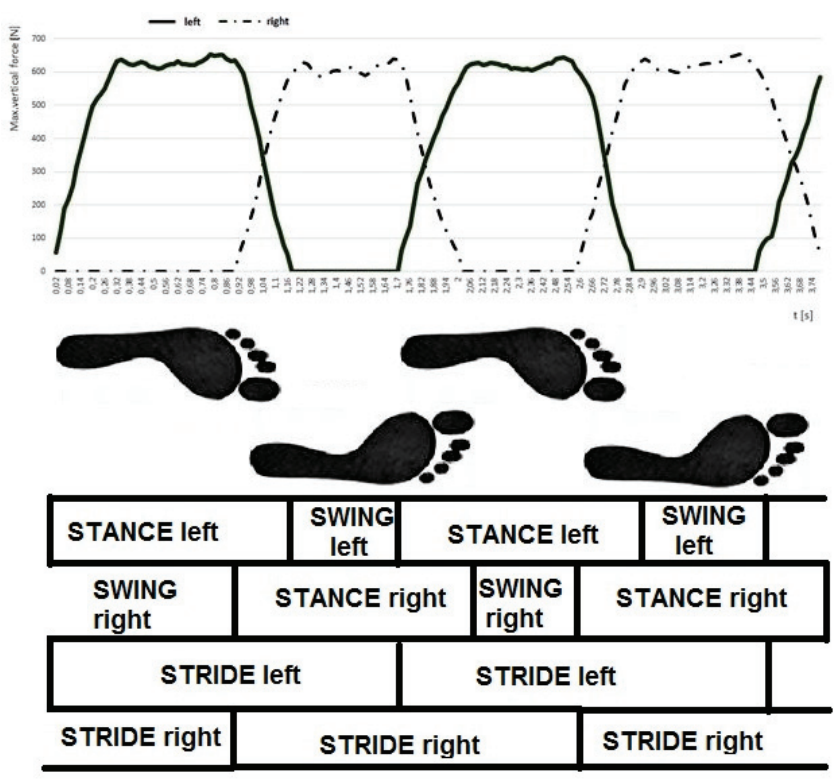

Fig. 2. An example of maximal vertical force component during the gait-cycle phases

\section{Posture analysis}

The results pertained to the posture in the sagittal and frontal planes, and the following values were compared: median sagittal bending index (mSBI), median frontal bending index (mFBI), sagittal bending amplitude (SBA), and the frontal bending amplitude (FBA) (Fig. 3) in all sections of the spine - lumbar (LSC), thoracic (TSC) and cervical (CSC). In the sagittal 
plane, the positive deviation represents flexion, while the negative one represents extension (Fig. 3). In the frontal plane, the positive values represent movement to the right, while negative ones represent movement to the left. These variables were compared for both types of shoes and subsequently statistically evaluated.
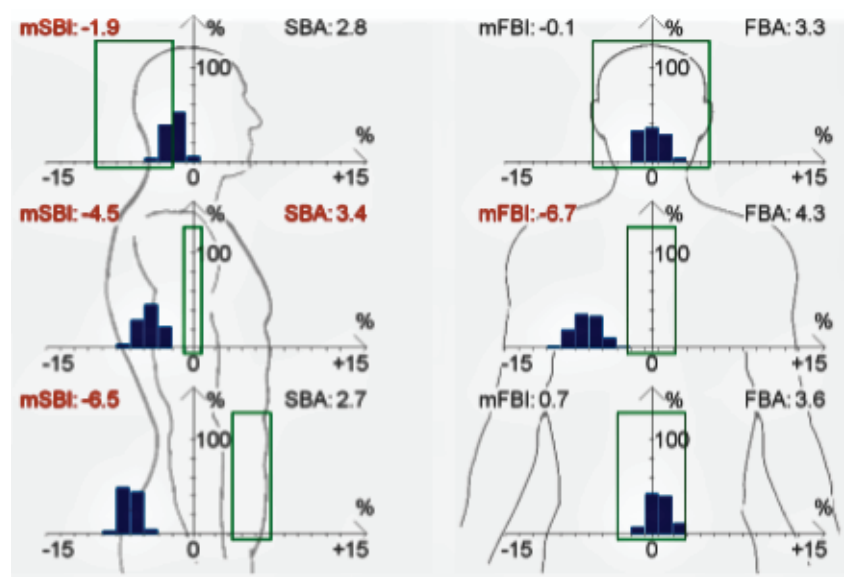

Fig. 3. Bodygram in the sagittal and frontal planes during walking in $\mathrm{HH}$ (The values outside the field of reference

- the standard is determined based on the calibration during upright posture - are provided in red)

\section{Statistical analysis}

The ANOVA statistical test with repeated measures $(\alpha=0.05)$ was used to evaluate the effect of $\mathrm{HH}$ on $P P_{\operatorname{maxBW}}, F_{\operatorname{maxBW}}$ and temporal variables of the gait cycle by comparing walking in $\mathrm{HH}$ and FS in $v_{1}, v_{2}$. The measured values were evaluated by comparing the calculated testing criterion of critical value $F$ - division (4.006873), where $F>F$ critical means that the hypothesis cannot be rejected. In the case of posture and for comparing differences in measured values depending on walking speed, a paired $t$-test was used as the statistical processing method to evaluate the interval data between the two dependent variables (i.e., paired data). The results are statistically significant if the $t$ value exceeds the critical table value of 1.669. The measured values are considered statistically significant at $(p \leq .05)$.

\section{Results}

\section{Temporal and plantar pressure characteristics of the gait cycle in HH and FS}

Significant differences in slow walking in $\mathrm{HH}$ and FS were detected in temporal variables (Table 1). For $v_{1}$, the STANCE duration was significantly shorter $(p=0.009)$ than in FS. The same trend was observed for $v_{2}(p=0.005)$. The SWING phase was significantly shorter while walking at $v_{1}(p=0.001)$. A difference in the SWING phase duration between FS and HH was not observed in $v_{2}$. In addition, STRIDE was significantly

Table 1. Differences in the plantar pressure distribution and the phases of the gait-cycle while walking slowly $\left(v_{1}\right)$ and very slowly $\left(v_{2}\right)$ in HH and FS

\begin{tabular}{|l|c|c|c|c|c|}
\hline $\begin{array}{c}\text { Temporal and Dynamic } \\
\text { Parameters in Plantar } \\
\text { Pressure Distribution }\end{array}$ & $\begin{array}{c}\text { FS } \\
\text { Mean SD }\end{array}$ & & $\begin{array}{c}\text { HH } \\
\text { Mean SD }\end{array}$ & & $\begin{array}{c}\text { FS vs. HH } \\
p \text {-value }\end{array}$ \\
\hline \multicolumn{1}{|c|}{$v_{1}$} & & Ratio [\%] & & Ratio [\%] & \\
\hline STANCE & $0.828 \pm 0.077$ & 64 & $0.780 \pm 0.058$ & 64 & $\mathbf{0 . 0 0 9}$ \\
\hline SWING & $0.476 \pm 0.046$ & 36 & $0.438 \pm 0.040$ & 36 & $\mathbf{0 . 0 0 1}$ \\
\hline STRIDE & $1.304 \pm 0.115$ & 100 & $1.217 \pm 0.084$ & 100 & $\mathbf{0 . 0 0 2}$ \\
\hline FRE & $92.468 \pm 8.392$ & & $99.004 \pm 6.597$ & & $\mathbf{0 . 0 0 2}$ \\
\hline$P P_{\operatorname{maxBW}}$ & $0.565 \pm 0.151$ & & $0.801 \pm 0.2$ & & $<\mathbf{0 . 0 0 1}$ \\
\hline$F_{\operatorname{maxBW}} v_{2}$ & $1.099 \pm 0.066$ & & $1.279 \pm 0.162$ & & $<\mathbf{0 . 0 0 1}$ \\
\hline & & Ratio [\%] & & Ratio $[\%]$ & \\
\hline STANCE & $1.215 \pm 0.115$ & 66 & $1.124 \pm 0.121$ & 66 & $\mathbf{0 . 0 0 5}$ \\
\hline SWING & $0.613 \pm 0.082$ & 34 & $0.580 \pm 0.064$ & 34 & 0.101 \\
\hline STRIDE & $1.829 \pm 0.179$ & 100 & $1.700 \pm 0.154$ & 100 & $\mathbf{0 . 0 0 5}$ \\
\hline FRE & $66.303 \pm 6.547$ & & $70.878 \pm 7.352$ & & $\mathbf{0 . 0 1 5}$ \\
\hline$P P_{\operatorname{maxBW}}$ & $0.535 \pm 0.142$ & & $0.762 \pm 0.192$ & & $<\mathbf{0 . 0 0 1}$ \\
\hline$F_{\operatorname{maxBW}}$ & $1.080 \pm 0.062$ & & $1.231 \pm 0.135$ & & $<\mathbf{0 . 0 0 1}$ \\
\hline
\end{tabular}

Legend: $P P_{\operatorname{maxBW}}-$ maximal peak pressure per body weight, $F_{\operatorname{maxBW}}-$ maximal vertical force per body weight, $\mathrm{HH}$ - high-heeled shoes, FS - flat shoes, SD - standard deviation, FRE - frequency per minute, $v_{1}$ - walking speed of $0.97 \mathrm{~m} \cdot \mathrm{s}^{-1}$ and $v_{2}-$ walking speed of $0.56 \mathrm{~m} \cdot \mathrm{s}^{-1}$ 
shorter in $\mathrm{HH}$ at $v_{1}(p=0.002)$ and also at $v_{2}(p=$ 0.005 ). The percentage ratio of the average duration pertaining to individual gait phases (STANCE, SWING) was equal in both types of shoes. At $v_{1}$, the ratio was $64 \%$ of STANCE versus $36 \%$ of SWING in $\mathrm{HH}$ and FS. For $v_{2}$ the STANCE phase was longer $(66 \%)$ and the SWING shorter $(34 \%)$ in both types of shoes. FRE was significantly higher in $\mathrm{HH}$ at $v_{1}(p=$ $0.002)$ and also at $v_{2}(p=0.015)$.

Significant differences between HH and FS were detected also in dynamic parameters $P P_{\operatorname{maxBW}}$ and $F_{\text {maxBw }}$ (Table 1), when the values of $P P_{\operatorname{maxBw}}$ and $F_{\text {maxBW }}$ were significantly higher in $\mathrm{HH}(p \leq .001)$.

Posture and spine changes

influenced by walking in $\mathrm{HH}$ and in FS

The evaluation of the overall results showed significant differences between walking in $\mathrm{HH}$ and FS in some of the measured parameters (Table 2). In the sagittal plane, significant differences in all sections of the spine at both measured walking speeds were identified. In the case of SBA, only at $v_{2}$ were significant differences found in LSC $(p=0.019)$, TSC $(p=0.003)$ and CSC $(p=0.002)$ (Table 2). Based on the overall results in the sagittal plane, it can be stated that $\mathrm{HH}$ affect the spine curvature, unlike FS. While walking in $\mathrm{HH}$, at $v_{1}$ and $v_{2}$ lordosis at LSC decreases significantly $(p<0.001)$ as well as CSC $(p<0.001)$, and kyphosis increases in TSC $(p<0.001)$ (Table 2).

In the frontal plane, statistically significant differences were observed only at $v_{1}$ in LSC $(p=0.013)$. In the frontal plane, the $v_{2}$ speed caused significant differences between HH and FS in CSC $(p=0.044)$ and movement amplitudes (FBA), in CSC $(p=0.002)$ and $\operatorname{LSC}(p=0.002)$.

Table 2. Differences in the individual spine section during walking in $\mathrm{HH}$ and FS

\begin{tabular}{|c|c|c|c|}
\hline $\begin{array}{c}\text { Parameters of Individual } \\
\text { Spine Section }\end{array}$ & $\begin{array}{c}\text { FS } \\
\text { Mean SD }\end{array}$ & $\begin{array}{c}\mathrm{HH} \\
\text { Mean SD }\end{array}$ & $\begin{array}{c}\text { FS vs. HH } \\
p \text {-value }\end{array}$ \\
\hline \multicolumn{4}{|l|}{$v_{1}$} \\
\hline $\mathrm{mSBI}-\mathrm{LSC}[\mathrm{mm}]$ & $-0.487 \pm 7.229$ & $0.550 \pm 6.962$ & $<0.001$ \\
\hline $\mathrm{SBA}-\mathrm{LSC}[\mathrm{mm}]$ & $2.503 \pm 1.075$ & $2.947 \pm 1.174$ & 0.112 \\
\hline $\mathrm{mSBI}-\mathrm{TSC}[\mathrm{mm}]$ & $-3.403 \pm 2.586$ & $-3.000 \pm 2.673$ & $<0.001$ \\
\hline $\mathrm{SBA}-\mathrm{TSC}[\mathrm{mm}]$ & $1.590 \pm 0.762$ & $1.687 \pm 0.850$ & 0.620 \\
\hline $\mathrm{mSBI}-\mathrm{CSC}[\mathrm{mm}]$ & $-4.120 \pm 5.019$ & $-2.240 \pm 4.392$ & $<0.001$ \\
\hline $\mathrm{SBA}-\mathrm{CSC}[\mathrm{mm}]$ & $4.850 \pm 1.994$ & $4.720 \pm 1.841$ & 0.606 \\
\hline $\mathrm{mFBI}-\mathrm{LSC}[\mathrm{mm}]$ & $-2.023 \pm 4.129$ & $-2.98 \pm 4.621$ & 0.013 \\
\hline FBA - LSC [mm] & $3.890 \pm 1.757$ & $4.410 \pm 1.555$ & 0.083 \\
\hline $\mathrm{mFBI}-\mathrm{TSC}[\mathrm{mm}]$ & $-1.686 \pm 3.417$ & $-1.376 \pm 3.716$ & 0.461 \\
\hline $\mathrm{FBA}-\mathrm{TSC}[\mathrm{mm}]$ & $1.843 \pm 1.160$ & $2.336 \pm 2.085$ & 0.792 \\
\hline $\mathrm{mFBI}-\mathrm{CSC}[\mathrm{mm}]$ & $-0.44 \pm 2.917$ & $-0.173 \pm 2.810$ & 0.067 \\
\hline FBA - CSC [mm] & $3.656 \pm 1.635$ & $3.770 \pm 1.905$ & 0.264 \\
\hline \multicolumn{4}{|l|}{$v_{2}$} \\
\hline $\mathrm{mSBI}-\mathrm{LSC}[\mathrm{mm}]$ & $0.003 \pm 6.626$ & $3.337 \pm 7.549$ & $<0.001$ \\
\hline SBA - LSC [mm] & $3.303 \pm 1.075$ & $4.140 \pm 2.247$ & 0.019 \\
\hline $\mathrm{mSBI}-\mathrm{TSC}[\mathrm{mm}]$ & $-2.147 \pm 2.134$ & $-0.770 \pm 2.169$ & $<0.001$ \\
\hline $\mathrm{SBA}-\mathrm{TSC}[\mathrm{mm}]$ & $1.733 \pm 0.727$ & $2.520 \pm 1.285$ & 0.003 \\
\hline $\mathrm{mSBI}-\mathrm{CSC}[\mathrm{mm}]$ & $-3.123 \pm 3.148$ & $-0.240 \pm 3.887$ & $<0.001$ \\
\hline $\mathrm{SBA}-\mathrm{CSC}[\mathrm{mm}]$ & $4.627 \pm 2.064$ & $5.853 \pm 2.391$ & 0.002 \\
\hline $\mathrm{mFBI}-\mathrm{LSC}[\mathrm{mm}]$ & $-2.893 \pm 3.811$ & $-2.76 \pm 3.845$ & 0.687 \\
\hline FBA - LSC [mm] & $4.277 \pm 2.039$ & $4.833 \pm 2.191$ & 0.002 \\
\hline $\mathrm{mFBI}-\mathrm{TSC}[\mathrm{mm}]$ & $-1.513 \pm 3.531$ & $-1.036 \pm 3.193$ & 0.086 \\
\hline FBA - TSC [mm] & $2.283 \pm 1.190$ & $3.140 \pm 1.434$ & 0.834 \\
\hline $\mathrm{mFBI}-\mathrm{CSC}[\mathrm{mm}]$ & $-0.35 \pm 2.436$ & $0.203 \pm 3.291$ & 0.044 \\
\hline FBA - CSC [mm] & $3.640 \pm 1.809$ & $3.590 \pm 1.654$ & 0.002 \\
\hline
\end{tabular}

Legend: LSC - lumbar spine, TSC - thoracic spine, CSC - cervical spine, mSBI - median Sagittal Bending Index, mFBI - median Frontal Bending Index, SBA - Sagittal Bending Amplitude, FBA - Frontal Bending Amplitude, $\mathrm{HH}$ - high-heeled shoes, FS - flat shoes, SD - standard deviation, $v_{1}$ - walking speed of $0.97 \mathrm{~m} \cdot \mathrm{s}^{-1}$ and $v_{2}-$ walking speed of $0.56 \mathrm{~m} \cdot \mathrm{s}^{-1}$ 


\section{Effect of walking speed}

The walking speed affected all measured temporal variables - STANCE, STRIDE, SWING, frequency per minute in both FS and HH $(p<0.001)$ (Table 3$)$.

In terms of posture, the walking speed affected the movement amplitudes of LSC in the sagittal plane (SBA) in FS $(p=0.006)$ and $\mathrm{HH}(p=0.013)$. In HH, a statistically significant differences between the walking speeds in TSC $-\operatorname{mSBI}(p=0.001)$ and SBA $(p=0.004)$ were observed (Table 3$)$.

Table 3. Differences in the measured values depending on the walking speed

\begin{tabular}{|l|c|c|}
\hline \multicolumn{1}{|c|}{$\begin{array}{c}\text { Measured } \\
\text { parameters }\end{array}$} & $\begin{array}{c}\text { FS }\left(v_{1}\right) \text { vs. FS }\left(v_{2}\right) \\
p \text {-value }\end{array}$ & $\begin{array}{c}\text { HH }\left(v_{1}\right) \text { vs. HH }\left(v_{2}\right) \\
p \text {-value }\end{array}$ \\
\hline STANCE & $<\mathbf{0 . 0 0 1}$ & $<\mathbf{0 . 0 0 1}$ \\
\hline STRIDE & $<\mathbf{0 . 0 0 1}$ & $<\mathbf{0 . 0 0 1}$ \\
\hline SWING & $<\mathbf{0 . 0 0 1}$ & $<\mathbf{0 . 0 0 1}$ \\
\hline FRE & $<\mathbf{0 . 0 0 1}$ & $<\mathbf{0 . 0 0 1}$ \\
\hline$P P_{\operatorname{maxBW}}$ & 0.441 & 0.441 \\
\hline$F_{\operatorname{maxBW}}$ & 0.275 & 0.215 \\
\hline mSBI - LSC $[\mathrm{mm}]$ & 0.788 & 0.143 \\
\hline SBA - LSC $[\mathrm{mm}]$ & $\mathbf{0 . 0 0 6}$ & $\mathbf{0 . 0 1 3}$ \\
\hline mSBI - TSC $[\mathrm{mm}]$ & $\mathbf{0 . 0 4 5}$ & $\mathbf{0 . 0 0 1}$ \\
\hline SBA - TSC $[\mathrm{mm}]$ & 0.459 & $\mathbf{0 . 0 0 4}$ \\
\hline mSBI - CSC $[\mathrm{mm}]$ & 0.302 & 0.061 \\
\hline mFBI - LSC $[\mathrm{mm}]$ & 0.400 & 0.842 \\
\hline FBA - LSC $[\mathrm{mm}]$ & 0.435 & 0.392 \\
\hline mFBI - TSC $[\mathrm{mm}]$ & 0.847 & 0.705 \\
\hline FBA - TSC $[\mathrm{mm}]$ & 0.152 & 0.087 \\
\hline mFBI - CSC $[\mathrm{mm}]$ & 0.897 & 0.635 \\
\hline FBA - CSC $[\mathrm{mm}]$ & 0.970 & 0.697 \\
\hline
\end{tabular}

Legend: $P P_{\operatorname{maxBW}}$ - maximal peak pressure per body weight, $F_{\text {maxBW }}$ - maximal vertical force per body weight, $\mathrm{HH}$ - high-heeled shoes, FS - flat shoes, LSC - lumbar spine, TSC - thoracic spine, CSC - cervical spine, mSBI - median Sagittal Bending Index, mFBI - median Frontal Bending Index, SBA - Sagittal Bending Amplitude, FBA - Frontal Bending Amplitude, SD - standard deviation, $v_{1}$ - walking speed of $0.97 \mathrm{~m} \cdot \mathrm{s}^{-1}$ and $v_{2}$ - walking speed of $0.56 \mathrm{~m} \cdot \mathrm{s}^{-1}$

\section{Discussion}

Wearing high-heeled shoes is a wide-spread model of behaviour among women in developed countries all over the world. The presented study enhances the knowledge pertaining to the effects of slow and very slow walking in $\mathrm{HH}$ observed in a group of inexperienced $\mathrm{HH}$ wearers; the walking speed was set to $0.97 \mathrm{~m} \cdot \mathrm{s}^{-1}$ and to $0.56 \mathrm{~m} \cdot \mathrm{s}^{-1}$, respectively. Both speeds were chosen intentionally based on the fact that higher speeds used in previous studies [2], [26] were perceived as too fast by the inexperienced $\mathrm{HH}$ wearers (subjects). Our subjects perceived the speeds selected for this study as comfortable.

According to the previous study, [10] the STANCE phase takes up about $60 \%$ of a single gaitcycle, while the remaining $40 \%$ pertain to the SWING phase. This study discovered that this ratio is influenced by the walking speed rather than by the type of shoes. At $v_{1}$, the STANCE phase amounted to $64 \%$ and SWING to $36 \%$ of the gait cycle in both $\mathrm{HH}$ and FS. At the very slow speed $v_{2}$, STANCE amounted to $66 \%$ and SWING to $34 \%$ of the gait cycle and it was identical in both types of shoes. This contradicts the previous study [16] which found that $\mathrm{HH}$ prolongs STANCE and shortens SWING, generally shortening the STRIDE in comparison with walking in FS, however, the cadence remained unaffected. While at comfortable and fast speeds the swinging leg moves along a largely ballistic trajectory under passive gravitational control, at significantly reduced walking speeds, a more active mode of control may be necessary to counteract gravity and provide sufficient 'air time' for the swinging leg [22]. Besides, the study showed that HH shortened both phases, which corresponds with previous studies [2], [7], [16]; cadence in $\mathrm{HH}$ increased in comparison with FS, which resulted from the fact that the treadmill was set to a constant speed.

The observation of plantar pressure distribution showed that $\mathrm{HH}$ increase the pressure variables $\left(P P_{\operatorname{maxBW}}, F_{\operatorname{maxBW}}\right)$ at both speeds, in comparison with FS. This also corresponds with previous studies [3], [4], [30]. At the slower speed, the duration of pressure is prolonged [31]; at the very slow speed $\mathrm{v}_{2}$, no differences in pressure values were recorded in comparison with $v_{1}$ in either shoes. In $\mathrm{HH}$, the pressure force was shifted forward and increased the forefoot pressure, which corresponds with other studies [4], [11], [30]. It indicates that a weight transfer mechanism is triggered by $\mathrm{HH}[1]$.

In accordance with previous studies [1], [15], significant differences were observed as a result of $\mathrm{HH}$ wearing - lumbar lordosis was straightened. Lumbar as well as cervical lordosis were decreased with statistical significance in $\mathrm{HH}$. However, there are also studies that discovered no significant differences in lordosis in HH [25]. The studies [20], [16] that report increased lordosis in $\mathrm{HH}$ were based on very small subject groups, for which they were criticised [6], [24]. Decreased lordosis in the lumbar area in inexperienced wearers and increased lordosis in experienced HH wearers were also reported [19]. There are even studies describing increased lordosis in inexperienced 
wearers [8], [21]. A study that measured the radiological parameters of the spine in the sagittal plane in $\mathrm{HH}$ and barefoot [27] claims that the differences in $\mathrm{C} 7$ and meatus vertical axis, cervical and lumbar lordosis, thoracic kyphosis, spino-sacral angle, pelvic tilt, sacral slope, and spinal tilt were not significant. Individuals adapting with less-than-average knee flexion responded to high heels by an additional increase in cervical lordosis. It may be explained by the fact that some subjects responded to high heels through their lower limbs, while some compensated the changed CoG by increased cervical lordosis. However, our study confirmed significant differences in all measured spine sections in the sagittal plane in $\mathrm{HH}$, since at both slow walking speeds both lumbar and cervical lordosis straightened. In the thoracic area, the kyphosis was increased in $\mathrm{HH}$. Moreover, at $v_{2}$ significant differences in amplitude values in $\mathrm{HH}$ were observed, which indicates major deviations in gait and instability caused by very slow walking speed. Different results in comparison with the previous study [27] can be ascribed to a different protocol (walking on the treadmill, constant walking speed, heel size). During walking, individual body segments move as well as the centre of gravity in response to the lower limb mechanics. As a result, a sinusoidal curve is created with a characteristic course in the given plane, best approximating the correct gait stereotype [30]. HH disrupt the stereotype causing the gait in $\mathrm{HH}$ to lose fluency [26]. Multiple authors claim that HH effects depend upon the individual's experience with this type of footwear [27], [19], [24], [29]. The results of this study indicate that the walking speed in $\mathrm{HH}$ can play an important role in the chain reaction of different kinematic effects influencing the posture.

However, this study also had several limitations, e.g., the subjects walked on a treadmill, which might have influenced their gait cycle. In addition, it should be noted that the walking speeds were constant for all subjects without respect to their individual anthropometric characteristics, although this setting allowed for recording of data under homogeneous conditions.

\section{Conclusions}

$\mathrm{HH}$ cause changes in the temporal and dynamic parameters of the gait cycle at slow walking speed. HH shorten both STANCE and STRIDE phases at speeds $v_{1}=0.97 \mathrm{~m} \cdot \mathrm{s}^{-1}$ and $v_{2}=0.56 \mathrm{~m} \cdot \mathrm{s}^{-1}$. The SWING phase was shortened only at $v_{1}$. HH also contribute to specific postural changes while walking. In
$\mathrm{HH}$, the lumbar and cervical spine lordosis were decreased in the sagittal plane, while kyphosis was increased in thoracic spine at both speeds. In the frontal plane, left lateral flexion in lumbar spine was observed in $\mathrm{HH}$ at $v_{1}$. At the slower speed $v_{2}$, the movement amplitude in lumbar spine increased in the frontal plane, while in cervical spine, the movement amplitude decreased and right lateral flexion occurred. At the slower walking speed in $\mathrm{HH}$, major movement amplitude changes occurred in the thoracic section of the spine.

Based on these results, it is recommended for inexperienced $\mathrm{HH}$ wearers to focus on the correct spine position while walking in $\mathrm{HH}$ and in situations that require a very slow walking speed, to consider not wearing $\mathrm{HH}$.

\section{Acknowledgement}

This work was supported by SGS 21290, Specific University Research Grant provided by the Ministry of Education, Youth and Sports of the Czech Republic.

\section{References}

[1] BAaklini E., ANGst M., Schellenberg F., Hitz M., Schmid S., TAL A., TAYLOR W.R., LORENZETTI S., High-heeled walking decreases lumbar lordosis, Gait Posture, 2017, 55, 12-14.

[2] Barkema D.D., Derrick T.R., Martin P.E., Heel height affects lower extremity frontal plane joint moments during walking, Gait Posture, 2012, 35, 438-488.

[3] Burnfield J.M., Few C.D., Mohamed O.S., Perry J., The influence of walking speed and footwear on plantar pressures in older adults, Clin. Biomech., 2004, 19, 78-84.

[4] Cong Y., Cheung J.T., Leung A.K., Zhang M., Effect of heel hight on in-shoe localized triaxial stresses, J. Biomech., 2011, 44, 2267-2272.

[5] Cowley E.E., Chevaller T.L., Chockallngam N., The effect of heel height on gait and posture, J. Am. Podiatr. Med. Assoc., 2009, 99, 512-518.

[6] CRONIN N.J., The effects of high-heeled shoes on female gait: A review, J. Electromyogr. Kines., 2014, 24, 258-263.

[7] Cronin N.J., BarretT R.S., CARTY C.P., Long-term use of high-heeled shoes alters the neuromechanics of human walking, J. Appl. Physiol., 2012, 112, 1054-1058.

[8] Dai M., Li X., Zhou X., Hu Y., LuO Q., ZHou S., High-heeled related alterations in the static sagittal profile of the spino-pelvic structure in young women, Eur. Spine J., 2015, 24, 1274-1281.

[9] Esenyel M., Walsh K., Walden J.G., Gitter A., Kinetics of high-heeled gait, J. Am. Podiatr. Med. Assoc., 2003, 93, 27-32.

[10] Gross J., Fetto J., Rosen E., Musculoskeletal Examination, third ed., Wiley-Blackwell, Oxford 2009.

[11] Hong W.H., Lee Y.H., Chen H.C., Pei Y.C., Wu C.Y., Influence of heel height and shoe insert on comfort perception and biomechanical performance of young female adults during walking, Foot Ankle Int., 2005, 26, 1042-1048. 
[12] CHIEN H.L., Lu T.W., LiU M.W., Effects of long-term wearing of high-heeled shoes on the central of the body's center of mass motion in relation to the center of pressure during walking, Gait Posture, 2014, 39, 1045-1050.

[13] Jandová S., Pazour J., Janura M., Comparison of Foot Load in walking after two different surgical treatment of calcaneal fracture, J. Foot Ankle Surg., 2019, 58, 260-265.

[14] Jandová S., ŠEflová I., Porovnáni zatiženi nohy v oblasti pod patni kostí při stoji v MBT obuvi a běžné sportovni obuvi, Rehabilitácia, 2015, 52, 49-56.

[15] Kramers-DE Quervain L.A., AtTinger BenZ D., MŸLler R., STŸSI E., Trunk and pelvic postural adaptation during high-heeled gait, Gait Posture, 1996, 4, 196-197.

[16] Lee C.M., Jeong E.H., Freivalds A., Biomechanical effects of wearing high-heeled shoes, Int. J. Ind. Ergon., 2001, 28, 321-326.

[17] LuXIMON Y., CONG Y., LuXIMON A., ZHANG M., Effects of heel base size, walking speed and slope angle on center of pressure trajectory and plantar pressure when wearing high-heeled shoes, Hum. Mov. Sci., 2015, 41, 307-319.

[18] Neumann P.A., Kinesiology of the musculoskeletal system: foundations for physical rehabilitation, third ed., Mosby, St. Louis, Missouri, 2002.

[19] Oliveira Pezzan P.A., Joao S.M., Ribeiro A.P., Manfio E.F., Postural assessment of lumbar lordosis and pelvic alingment angles in adolescent users and nonusers of high-heeled shoes, J. Manip. Physiol. Ther., 2011, 34, 614-621.

[20] OpILA-Correia K.A., Kinematics of high-heeled gait with consideration for age and experience of wearers, Arch. Phys. Med. Rehabil., 1990, 71, 905-909.

[21] Opila-Correia K.A., Kinematics of high-heeled gait, Arch. Phys. Med. Rehabil., 1990, 71, 304-309
[22] Otter A.R., Geurts A.C.H., Mulder T., Duysens J., Speed-related changes in muscle activity from normal to very slow walking speeds, Gait Posture, 2004, 9, 270-278.

[23] Perry J., Gait analysis: Normal and pathological functions, Slack Incorporated, New Jersey 1992.

[24] Russell B.S., MuhlenkAmp K.A., Hoirris K.T., DESIMONE C.M., Measurement of lumbar lordosis in static standing posture with and without high-heeled shoes, J. Chiropr. Med., 2012, 11, 145-153.

[25] Schroeder J., Hollander K., Effects of high-heeled footwear on static and dynamic pelvis position and lumbar lordosis in experienced younger and middle-aged women, Gait Posture, 2018, 59, 53-57.

[26] STEFAnyshyn D.J., NigG B.M., Fisher V., FlynN B.Ó, LiU W., The influence of high-heeled shoes on kinematics, kinetics, and muscle EMG of normal female gait, J. Appl. Biomech., 2000, 16, 309-319.

[27] Weitkunat T., Buck F.M., Jentzsch T., Simmen H.P., WERNER C.M., OSTERHOFF G., Influence of high-heeled shoes on the sagittal balance of the spine and the whole body, Eur. Spine J., 2016, 25, 3658-3665

[28] Whittle M., Gait analysis: an introduction, Butterworth Heinemann - Elsevier, Edinburg 2007.

[29] Winiarski S., RutKowska-Kucharska A., Zostawa P., Uścinowicz-Zostawa N., Klichet S., Foot mechanics in young women are altered after walking in high-heeled shoes, Acta Bioeng. Biomech., 2017, 19, 107-113.

[30] YunG-Hui L., WeI-HsIEN H., Effects of shoe inserts and heel height on foot pressure, impact force, and perceived comfort during walking, Appl. Ergon., 2005, 36, 355-362.

[31] Zhu H.S., Wertsch J.J., HARRIS G.F., LOFTSGAARDEN J.D., PRICE M.B., Foot pressure distribution during walking and shuffling, Arch. Phys. Med., 1991, 72, 390-397. 